\title{
Comparison of Rough-set and Interval-set Models for Uncertain Reasoning
}

\author{
Y.Y. Yao and Xining Li \\ Department of Computer Science \\ Lakehead University \\ Thunder Bay, Ontario, Canada P7B 5E1
}

\begin{abstract}
In the rough-set model, a set is represented by a pair of ordinary sets called the lower and upper approximations. In the interval-set model, a pair of sets is referred to as the lower and upper bounds which define a family of sets. A significant difference between these models lies in the definition and interpretation of their extended set-theoretic operators. The operators in the rough-set model are not truth-functional, while the operators in the interval-set model are truth-functional. Within the framework of possible-worlds analysis, we show that the rough-set model corresponds to the modal logic system $\mathrm{S}_{5}$, while the interval-set model corresponds to Kleene's three-valued logic system $\mathrm{K}_{3}$. It is argued that these two models extend set theory in the same manner as the logic systems $\mathrm{S}_{5}$ and $\mathrm{K}_{3}$ extend standard propositional logic. Their relationships to probabilistic reasoning are also examined.
\end{abstract}

\section{Introduction}

The rough-set and interval-set models are two related but distinct extensions of set theory for modeling vagueness. In the rough-set model, a given set is represented by a pair of ordinary sets called the lower and upper approximations [21,22]. The approximation space is constructed based on an equivalence relation defined by a set of attributes. There are two views for the interpretation of the rough-set model. Under one view, the approximation space can be understood in terms of two additional settheoretic operators [12]. They assign for each subset of the universe a lower approximation and an upper approximation [21]. We regard this interpretation to be the operator-oriented view. The other interpretation is a set-oriented view which considers a rough set as the family of sets having the same lower and upper approximations $[2,3]$. The rough-set model is useful in the study of information system, classification and machine learning [24]. In the interval-set model, it is assumed that the available information is insufficient to define a set precisely [36,37]. Instead, a pair of sets referred to as the lower and upper bounds is used to define the range of the unknown set. In other words, any member of the family of sets bounded by the lower and upper bounds can in fact be the set. Such a framework is similar to the interval-numeric algebra [1, 14].

There have been extensive studies on the logical foundation of these extensions of set theory and their relationship to non-standard logics [23, 24, 25, 26, 27]. For example, Orlowska proposed a logic for reasoning about concepts using the notion of rough sets, which is essentially the modal logic system $S_{5}$ with the modal operators interpreted using the lower and upper approximations [19]. A similar approach was also adopted by Chakraborty and Banerjee [5]. Vakarelov considered the lower and upper approximations formed from different types of relations as additional and distinct modal operators [35]. The semantics of these logic systems have been investigated by many authors [15, 20]. Recently, Yao and Li examined the relationship between the interval-set model and Kleene's threevalued logic [37]. It has been shown that the interval-set model provides the possible-worlds semantics for Kleene's three-valued logic.

Based on the above studies, this paper provides a comparison of rough-set and interval-set models with emphasis on uncertain reasoning. The discussion will focus on the interpretation of these models and their connections and differences. The main objective of such a comparative study is to show that these two models provide different and complementary extensions of set theory, although both use a pair of sets in their formulations. More importantly, they extend set theory in the same way 
that modal and three-valued logics extend propositional logic. The roles played by both models in uncertainty management are illustrated through probabilistic reasoning.

This paper is an expanded version of our earlier paper [37] and is organized as follows. Section 2 reviews the key concepts of the rough-set and interval-set models and examines their interpretations and algebraic differences. Section 3 summarizes the possible-worlds analysis approaches, which demonstrates the connection between set theory and two-valued propositional logic. Section 4 presents a possible-worlds analysis of modal logic system $\mathrm{S}_{5}$ based on the rough-set model. In the same manner, Section 5 presents a possible-worlds analysis of Kleene's three-valued logic system $\mathrm{K}_{3}$ using the interval-set model.

\section{Rough-set and Interval-set Models}

This section reviews and compares the rough-set and interval-set models from the view point of their algebraic structures. Both models are formulated based on a finite and non-empty set $U$ called the universe. The interpretation of the universe depends on the particular applications of the models.

In the rough set model, there is an equivalence relation $\Re$ defined on $U$, namely, $\Re$ is reflexive, symmetric and transitive. This relation partitions $U$ into disjoint subsets $U / \Re=\left\{E_{1}, E_{2}, \ldots, E_{n}\right\}$, where $E_{i}$ is an equivalence class of $\Re$. The family $U / \Re$ is called a quotient set and the pair apr $=(U, \Re)$ is called an approximation space. The empty set and the elements of $U / \Re$ are called the elementary or atomic sets. The union of one or more elementary sets is called a composed set. The family of all composed sets is denoted by $\operatorname{Com}(a p r)$.

The elementary sets are the building blocks for the construction of rough sets. In the approximation space $a p r=(U, \Re)$, given an arbitrary set $A \subseteq U$, one may represent $A$ by a pair of lower and upper approximations:

$$
\begin{aligned}
& \underline{\operatorname{apr}(A)}=\bigcup_{E_{i} \subseteq A} E_{i}=\left\{x \in U \mid[x]_{\Re} \subseteq A\right\}, \\
& \overline{\operatorname{apr}}(A)=\bigcup_{E_{i} \cap A \neq \emptyset} E_{i}=\left\{x \in U \mid[x]_{\Re} \cap A \neq \emptyset\right\},
\end{aligned}
$$

where $[x]_{\Re}$ denote the equivalence class containing $x$. The lower approximation $\operatorname{apr}(A)$ is the union of all the elementary sets which are subsets of $A$, and the upper approximation $\overline{\overline{a p r}}(A)$ is the union of all the elementary sets which have a non-empty intersection with $A$. In fact, $\operatorname{apr}(A)$ is the greatest composed set contained by $A$, while $\overline{a p r}(A)$ is the least composed set containing $A$. These two sets may be equivalently defined as:

$$
\begin{aligned}
& \underline{\operatorname{apr}}(A)=\{x \in U \mid \text { for all } y \in U, x \Re y \text { implies } y \in A\}, \\
& \overline{\operatorname{apr}}(A)=\{x \in U \mid \text { there exists a } y \in U \text { such that } x \Re y \text { and } y \in A\} .
\end{aligned}
$$

The pair $(\operatorname{apr}(A), \overline{\operatorname{apr}}(A))$ is called the rough set of $A$.

This interpretation of the rough-set model provides an operator-oriented view, in which apr and $\overline{a p r}$ are understood as a pair of additional unary set-theoretic operators [12]. The following list summarizes their properties pertinent to our discussion. For any subsets $A, B \subseteq U$ :

$$
\begin{aligned}
& \underline{\operatorname{apr}}(A) \subseteq A \subseteq \overline{\operatorname{apr}}(A), \\
& \overline{\operatorname{apr}}(\emptyset)=\overline{\operatorname{apr}}(\emptyset)=\emptyset \text {, } \\
& \overline{\operatorname{apr}}(U)=\overline{\operatorname{apr}}(U)=U \text {, } \\
& \underline{\operatorname{apr}}(A \cap B)=\underline{a p r}(A) \cap \underline{\operatorname{apr}}(B), \\
& \overline{a p r}(A \cap B) \subseteq \overline{a p r}(A) \cap \overline{a p r}(B) \text {, } \\
& \underline{\operatorname{apr}}(A \cup B) \supseteq \underline{\operatorname{apr}}(A) \cup \underline{\operatorname{apr}}(B), \\
& \overline{\overline{a p r}}(A \cup B)=\overline{\overline{a p r}}(A) \cup \overline{\overline{a p r}}(B) \text {, } \\
& \underline{\operatorname{apr}}(A)=\left(\overline{\operatorname{apr}}\left(A^{c}\right)\right)^{c}, \quad \overline{\operatorname{apr}}(A)=\left(\underline{\operatorname{apr}}\left(A^{c}\right)\right)^{c}, \\
& \operatorname{apr}(A)=\operatorname{apr}(\operatorname{apr}(A))=\overline{\operatorname{apr}}(\operatorname{apr}(A)), \\
& \overline{\overline{a p r}}(A)=\overline{\operatorname{apr}}(\overline{\overline{a p r}}(A))=\overline{a p r}(\overline{\overline{a p r}}(A)) .
\end{aligned}
$$

where $A^{c}=U-A$ denotes the set complement of $A$. Property (R1) says that the pair of operators produces a range within which lies the given set. Properties (R2) and (R3) are the conditions that the operators must meet at the two extreme points of $2^{U}$, the minimum element $\emptyset$ and the maximum 
element $U$. Properties (R4)-(R7) represent the weak distributivity and distributivity of operators $\underline{a p r}$ and $\overline{a p r}$. Property (8) states that the pair of operators are dual to each other. Properties (R9) and (R10) indicate that the result of a double application of the new operators is the same as that of a single application. It is important to note that these properties are not independent.

Consider now a special equivalence relation in which each equivalence class contains only a single element. From equation (1), it is clear that both $a p r$ and $\overline{a p r}$ become the identity operator, i.e., $\operatorname{apr}(A)=\overline{\operatorname{apr}}(A)=A$ for all $A \in 2^{U}$. In this case, it is not necessary to consider the effect of these $\overline{\text { two }}$ operators and the rough set $(\underline{\operatorname{apr}}(A), \overline{\operatorname{apr}}(A))$ reduces to the ordinary set $A$. From this standing point, one may consider the rough set model as an extension of set theory with two new set-theoretic operators.

In the interval-set model, given two sets $A_{1}, A_{2} \in 2^{U}$ with $A_{1} \subseteq A_{2}$, the following subset of $2^{U}$,

$$
\mathcal{A}=\left[A_{1}, A_{2}\right]=\left\{X \in 2^{U} \mid A_{1} \subseteq X \subseteq A_{2}\right\},
$$

is called a closed interval set. The set $A_{1}$ is called the lower bound of the interval set and $A_{2}$ the upper bound. That is, an interval set is a set of subsets bounded by two elements of the Boolean algebra $\left(2^{U}, \cap, \cup,{ }^{c}\right)$. Let $I\left(2^{U}\right)$ denote the set of all closed interval sets.

Let $\cap, \cup$ and - be the usual set intersection, union and difference defined on $2^{U}$, respectively. We define the following binary operations on interval sets based on the set-theoretic operations on their members. For two interval sets $\mathcal{A}=\left[A_{1}, A_{2}\right]$ and $\mathcal{B}=\left[B_{1}, B_{2}\right]$, the interval-set intersection, union and difference are defined as:

$$
\begin{aligned}
& \mathcal{A} \sqcap \mathcal{B}=\{X \cap Y \mid X \in \mathcal{A}, Y \in \mathcal{B}\}, \\
& \mathcal{A} \sqcup \mathcal{B}=\{X \cup Y \mid X \in \mathcal{A}, Y \in \mathcal{B}\}, \\
& \mathcal{A} \backslash \mathcal{B}=\{X-Y \mid X \in \mathcal{A}, Y \in \mathcal{B}\} .
\end{aligned}
$$

The above defined operators are closed on $I\left(2^{U}\right)$, namely, $\mathcal{A} \sqcap \mathcal{B}, \mathcal{A} \sqcup \mathcal{B}$ and $\mathcal{A} \backslash \mathcal{B}$ are interval sets. In fact, these interval sets can be explicitly computed by:

$$
\begin{aligned}
\mathcal{A} \sqcap \mathcal{B} & =\left[A_{1} \cap B_{1}, A_{2} \cap B_{2}\right], \\
\mathcal{A} \sqcup \mathcal{B} & =\left[A_{1} \cup B_{1}, A_{2} \cup B_{2}\right], \\
\mathcal{A} \backslash \mathcal{B} & =\left[A_{1}-B_{2}, A_{2}-B_{1}\right] .
\end{aligned}
$$

The interval-set complement $\neg\left[A_{1}, A_{2}\right]$ of $\left[A_{1}, A_{2}\right]$ is defined by $[U, U] \backslash\left[A_{1}, A_{2}\right]$. This is equivalent to $\left[U-A_{2}, U-A_{1}\right]=\left[A_{2}^{c}, A_{1}^{c}\right]$. Obviously, we have $\neg[\emptyset, \emptyset]=[U, U]$ and $\neg[U, U]=[\emptyset, \emptyset]$.

For operators $\sqcap, \sqcup$ and $\neg$, the following properties hold: for $\mathcal{A}, \mathcal{B}, \mathcal{C} \in I\left(2^{U}\right)$,

(I1) Idempotent :

$\mathcal{A} \sqcap \mathcal{A}=\mathcal{A}, \quad \mathcal{A} \sqcup \mathcal{A}=\mathcal{A} ;$

(I2) Commutativity :

$$
\mathcal{A} \sqcap \mathcal{B}=\mathcal{B} \sqcap \mathcal{A}, \quad \mathcal{A} \sqcup \mathcal{B}=\mathcal{B} \sqcup \mathcal{A} ;
$$

(I3) Associativity :

$$
\begin{aligned}
& (\mathcal{A} \sqcap \mathcal{B}) \sqcap \mathcal{C}=\mathcal{A} \sqcap(\mathcal{B} \sqcap \mathcal{C}), \\
& (\mathcal{A} \sqcup \mathcal{B}) \sqcup \mathcal{C}=\mathcal{A} \sqcup(\mathcal{B} \sqcup \mathcal{C}),
\end{aligned}
$$

(I4) Distributivity :

$$
\begin{aligned}
& \mathcal{A} \sqcap(\mathcal{B} \sqcup \mathcal{C})=(\mathcal{A} \sqcap \mathcal{B}) \sqcup(\mathcal{A} \sqcap \mathcal{C}), \\
& \mathcal{A} \sqcup(\mathcal{B} \sqcap \mathcal{C})=(\mathcal{A} \sqcup \mathcal{B}) \sqcap(\mathcal{A} \sqcup \mathcal{C}) ;
\end{aligned}
$$

(I5) Absorption :

$$
\mathcal{A} \sqcap(\mathcal{A} \sqcup \mathcal{B})=\mathcal{A}, \quad \mathcal{A} \sqcup(\mathcal{A} \sqcap \mathcal{B})=\mathcal{A} ;
$$

De Morgan's laws :

$$
\neg(\mathcal{A} \sqcap \mathcal{B})=\neg \mathcal{A} \sqcup \neg \mathcal{B}, \quad \neg(\mathcal{A} \sqcup \mathcal{B})=\neg \mathcal{A} \sqcap \neg \mathcal{B} ;
$$

Double negation law :

$\neg \neg \mathcal{A}=\mathcal{A}$,

$[U, U]$ and $[\emptyset, \emptyset]$ are the unique identities for interval-set

intersection and union, that is,

$\mathcal{A}=\mathcal{X} \sqcap \mathcal{A}=\mathcal{A} \sqcap \mathcal{X}$ for all $\mathcal{A} \in I\left(2^{U}\right) \Longleftrightarrow \mathcal{X}=[U, U]$,

$\mathcal{A}=\mathcal{Y} \sqcup \mathcal{A}=\mathcal{A} \sqcup \mathcal{Y}$ for all $\mathcal{A} \in I\left(2^{U}\right) \Longleftrightarrow \mathcal{Y}=[\emptyset, \emptyset]$. 
These properties may be regarded as the counterparts of the properties of the corresponding settheoretic operators. However, for an interval set $\mathcal{A}, \mathcal{A} \sqcap \neg \mathcal{A}$ is not necessarily equal to $[\emptyset, \emptyset], \mathcal{A} \sqcup \neg \mathcal{A}$ is not necessarily equal to $[U, U]$, and $\mathcal{A} \backslash \mathcal{A}$ is not necessarily equal to $[\emptyset, \emptyset]$. Nevertheless, the following properties hold:

$$
\begin{aligned}
& \emptyset \in \mathcal{A} \sqcap \neg \mathcal{A}, \\
& U \in \mathcal{A} \sqcup \neg \mathcal{A}, \\
& \emptyset \in \mathcal{A} \backslash \mathcal{A} .
\end{aligned}
$$

Therefore, $I\left(2^{U}\right)$ is a completely distributive lattice but not a Boolean algebra, whereas $2^{U}$ is a Boolean algebra [17].

The interval-set model can be considered as a set-theoretic counterpart of the interval-number algebra $[1,14]$. The interpretation of an interval set given by equation (3) provides plausible justification for the proposed interval-set operators. Moreover, degenerate interval sets of the form $[A, A]$ are equivalent to ordinary sets. For degenerate interval sets, the proposed operators $\sqcap, \sqcup, \backslash$, and $\neg$ reduce to the usual set-theoretic operators. Thus, interval-set model may be considered as an extension of set theory with extended set-theoretic operators.

From the above discussion, it is clear that rough-set and interval-set models are different extensions of set theory. The rough-set model introduces two additional set-theoretic operators based on an equivalence relation on the universe. It forms a system $\left(2^{U}, \cap, \cup,{ }^{c}, \underline{a p r}, \overline{a p r}\right)$ by adding a pair of unary operators to the Boolean algebra $\left(2^{U}, \cap, \cup,{ }^{c}\right)$. On the other hand, the interval-set model extends the standard set-theoretic operators $\cap, \cup$ and ${ }^{c}$. It establishes a completely distributive lattice $\left(I\left(2^{U}\right), \sqcap, \sqcup\right)$ from the Boolean algebra $\left(2^{U}, \cap, \cup,{ }^{c}\right)$. It should be noted that interval-set operators are truth-functional, namely, the value of a compound formula can be computed from its components. In contrast, the two new rough-set operators are not truth-functional. In the next two sections, it will be seen that these extensions of set theory correspond to two different extensions of propositional logic.

An interval set $\left[A_{1}, A_{2}\right]$ is also referred to as a flou set [17]. The lower bound $A_{1}$ defines the sure region, the upper bound $A_{2}$ defines the maximum region, and the difference $A_{2}-A_{1}$ defines the flou region. The sure region corresponds to the positive region of a rough set and the flou region corresponds to the doubtful region. From this point of view, the notion of interval sets is related to the concept of rough sets.

An alternative view of rough-set model along the same line of argument as that of the intervalset model has been discussed by Bonikowski $[2,3,21]$. It presents a set-oriented interpretation of rough-set model. Given two elements $A_{1}, A_{2} \in \operatorname{Com}(a p r)$ with $A_{1} \subseteq A_{2}$, a rough set is defined as the following family of subsets of $U$ :

$$
\left\langle A_{1}, A_{2}\right\rangle=\left\{X \in 2^{U} \mid \underline{\operatorname{apr}}(X)=A_{1}, \overline{\operatorname{apr}}(X)=A_{2}\right\} .
$$

Under this interpretation, one can extend the set-theoretic operators $\cap, \cup$ and ${ }^{c}$ into the corresponding rough-set operators: for two rough sets $\left\langle A_{1}, A_{2}\right\rangle$ and $\left\langle B_{1}, B_{2}\right\rangle$,

$$
\begin{aligned}
\left\langle A_{1}, A_{2}\right\rangle \otimes\left\langle B_{1}, B_{2}\right\rangle & =\left\{X \in 2^{U} \mid \underline{\operatorname{apr}}(X)=A_{1} \cap B_{1}, \overline{\operatorname{apr}}(X)=A_{2} \cap B_{2}\right\}, \\
\left\langle A_{1}, A_{2}\right\rangle \oplus\left\langle B_{1}, B_{2}\right\rangle & =\left\{X \in 2^{U} \mid \underline{\operatorname{apr}}(X)=A_{1} \cup B_{1}, \overline{\operatorname{apr}}(X)=A_{2} \cup B_{2}\right\}, \\
\ominus\left\langle A_{1}, A_{2}\right\rangle & =\left\{X \in 2^{U} \mid \underline{\operatorname{apr}}(X)=A_{2}^{c}, \overline{\operatorname{apr}}(X)=A_{1}^{c}\right\} .
\end{aligned}
$$

The symbols $\otimes, \oplus$ and $\ominus$ represent the rough-set intersection, union and complement. Unlike the interval-set operators, these rough-set operators are not truth-functional. Let $R($ apr $)$ denote the set of all rough sets in the approximation space apr. The extended system $(R(a p r), \otimes, \oplus)$ is a completely distributive lattice [3].

\section{Propositional Logic and Set Theory}

The possible-worlds semantics analysis has been used to interpret various approaches for uncertainty representation and uncertain reasoning, such as propositional logic, modal logic, probabilistic logic, and fuzzy logic $[4,9,18,30,31]$. This framework consists of, for example, a propositional language, a set of possible worlds, and a valuation function that maps each proposition to a truth value with respect to a particular possible world. Through the valuation function, one can map a proposition into a subset of possible worlds in which the proposition is true. The logical operators are therefore interpreted in terms of set-theoretic operators. If one defines a probability function on the set of all possible worlds, the true-false semantics for logical sentences is extended to a semantics that allows 
probabilistic values on sentences. In this section, a true-unknown-false semantics is also used in addition to the ordinary true-false semantics $[4,30,34]$.

Let $\Phi$ be a finite non-empty set of propositions of interest. A propositional language formed from $\Phi$ is denoted by $L(\Phi)$, which is the smallest set containing the members of $\Phi$ and two particular propositions $\perp$ and $T$, and is closed under negation $(\sim)$, conjunction $(\wedge)$, disjunction $(\vee)$, and implication $(\rightarrow)$. Let $W$ be a non-empty set of possible worlds, which is used to represent states or situations of the system being modeled. Each possible world can be considered as a partial interpretation of some logical formulas in the propositional language $L(\Phi)$. Formally, for a particular possible world $w$, we define a valuation function $\pi_{w}: L(\Phi) \longrightarrow\{$ true, false $\}$. If $\pi_{w}(\phi)=$ true, we say that the proposition $\phi$ is true in the world $w$, otherwise we say that the proposition is false.

Using valuation functions $\pi_{w}$ for all possible worlds, we can define a mapping $i: L(\Phi) \longrightarrow 2^{W}$ as follows:

$$
i(\phi)=\left\{w \in W \mid \pi_{w}(\phi)=\text { true }\right\} .
$$

The set $i(\phi)$ contains those possible worlds in which $\phi$ is true. It can be interpreted as the truth set of the proposition $\phi$. This mapping is also referred to as an incidence mapping and $i(\phi)$ the incidence of $\phi$ [4]. According to the semantics of two-valued logic, given two propositions $\phi$ and $\psi, \phi \wedge \psi$ is true in a world $w$ if and only if both $\phi$ and $\psi$ are true in $w$. Similar rules can be used for other logical connectives. In summary, an incidence mapping should satisfy the following properties:

$$
\begin{aligned}
& i(\perp)=\emptyset, \\
& i(\top)=W, \\
& i(\sim \phi)=(i(\phi))^{c}, \\
& i(\phi \wedge \psi)=i(\phi) \cap i(\psi), \\
& i(\phi \vee \psi)=i(\phi) \cup i(\psi), \\
& i(\phi \rightarrow \psi)=(i(\phi))^{c} \cup i(\psi) .
\end{aligned}
$$

Properties (i3)-(i6) indicate that the incidence mapping is truth-functional, which reflects the truthfunctional property of the logic connectives in propositional logic. An advantage of this framework is that the logical connectives $\wedge, \vee$, and $\sim$ are interpreted using the set-theoretic operators $\cap, \cup$ and ${ }^{c}$. One may therefore say that set theory provides an interpretation for propositional logic.

In practice, we only know the incidences of a subset of all propositions in $L(\Phi)$. Thus, an important task is to infer the incidences of these propositions whose incidences are not given. In propositional logic, the modus ponens rule:

$$
\frac{\phi \rightarrow \psi, \phi}{\psi}
$$

allows us to infer $\psi$ from $\phi \rightarrow \psi$ and $\phi$. In the framework of incidence calculus, it is impossible to derive the incidence $i(\psi)$ from $i(\phi \rightarrow \psi)$ and $i(\phi)$, using the above modus ponens rule. Instead, we may specify bounds within which lies the true incidence $i(\psi)$. Consider a world $w$ in which both $\phi \rightarrow \psi$ and $\phi$ are true, we can immediately conclude that $\psi$ is also true in $w$. That is, $w \in i(\phi) \cap i(\phi \rightarrow \psi)$ implies $w \in i(\psi)$. Similarly, if $\psi$ is true in a world $w$, then $\phi \rightarrow \psi$ is also true in $w$, namely, $w \in i(\psi)$ implies $w \in i(\phi \rightarrow \psi)$. However, it is impossible to have a world in which both $\phi$ and $\phi \rightarrow \psi$ are false, such a world is referred to as an inconsistent world. Nilsson proposed a method using binary semantic tree to perform such an analysis [18]. All consistent possible worlds are summarized in the following table:

\begin{tabular}{ccc}
$\phi \rightarrow \psi$ & $\phi$ & $\psi$ \\
\hline true & true & true \\
true & false & $\{$ true,false $\}$ \\
false & true & false
\end{tabular}.

The second row says that if $\phi \rightarrow \psi$ is true and $\phi$ is false in a world $w$, then $\psi$ can either be true or false in $w$. According to this table, the best bounds of $i(\psi)$ is given by the interval set $[i(\phi) \cap i(\phi \rightarrow$ $\psi), i(\phi \rightarrow \psi)]$, based solely on $i(\phi \rightarrow \psi)$ and $i(\phi)$. Therefore, the following modified modus ponens rule may be used in incidence calculus:

$$
\frac{i(\phi \rightarrow \psi), \quad i(\phi)}{i(\phi) \cap i(\phi \rightarrow \psi) \subseteq i(\psi) \subseteq i(\phi \rightarrow \psi)} .
$$


More inference rules using incidence bounds can be found in [4].

The incidence calculus can also be used for quantitative inference. Suppose there is a probability function $P_{0}$ defined on the set of possible worlds $W$. By setting the probability of a proposition as the probability of its incidence, i.e.,

$$
P(\phi)=P_{0}(i(\phi)),
$$

we can immediately perform probabilistic inference $[4,18,30]$. For example, based on the modus ponens rule (10), we have:

$$
P_{0}(i(\phi) \cap i(\phi \rightarrow \psi)) \leq P_{0}(i(\psi)) \leq P_{0}(i(\phi \rightarrow \psi)) .
$$

It can be equivalently expressed as:

$$
P(\phi \rightarrow \psi)+P(\phi)-1 \leq P(\psi) \leq P(\phi \rightarrow \psi),
$$

which are the bounds given by Nilsson [18].

\section{Modal Logic and Rough-set Model}

The modal propositional logic is an extension of propositional logic with a pair of additional logic operators, while the rough-set model is an extension of set theory with a pair of extra set-theoretic operators. There is a close connection between these two frameworks [5, 15, 19, 23].

In the modal propositional logic, each proposition still takes either true or false as its truth value. The standard logic connectives $\wedge, \vee, \sim$ and $\rightarrow$ have the same semantics as in propositional logic. On the other hand, the propositional language is extended by introducing a pair of necessity and possibility operators $\square$ and $\diamond$. Since these modal operators are not truth-functional, their semantics cannot be defined using truth tables. In defining the possible-worlds semantics of modal operators, it is assumed that there is a binary relation $\Re$ on the set of possible worlds $W[6,28]$. The binary relation $\Re$ is called an accessible relation, which describes the relationships between different possible worlds. For two possible worlds $w$ and $w^{\prime}$, if $w \Re w^{\prime}$, we say that $w^{\prime}$ is accessible from $w$. The truth values of $\square(\phi)$ and $\diamond(\phi)$ in a possible world $w$ are defined based on the truth values of the proposition $\phi$ in the possible worlds accessible from $w$ :

$$
\begin{aligned}
& \pi_{w}(\square \phi)=\text { true } \quad \text { iff } \quad \text { for all } w^{\prime} \in W, w \Re w^{\prime} \text { implies } \pi_{w^{\prime}}(\phi)=\text { true, } \\
& \pi_{w}(\diamond \phi)=\text { true } \quad \text { iff } \quad \text { there exists a } w^{\prime} \in W \text { such that } w \Re w^{\prime} \text { and } \pi_{w^{\prime}}(\phi)=\text { true. }
\end{aligned}
$$

That is, a proposition $\psi$ is necessarily true in a world $w$ if and only if it is true in all the worlds that are accessible from $w$; a proposition $\psi$ is possibly true in a world $w$ if and only if it is true in at least one of the worlds that are accessible from $w$. Obviously, $\square$ and $\diamond$ are dual operators, namely, $\diamond \phi=\sim \square \sim \phi$.

In the case where $\Re$ is an equivalence relation, one obtains the modal logic system $\mathrm{S}_{5}$. In this system, using the equivalence relation $\Re$ on $W$, one can construct an approximation space apr $=$ $(W, \Re)$. Let $L_{m}(\Phi)$ denote the modal propositional language that incorporates the modal operators in addition to the standard logic connectives. By equation (8), we can define a generalized incidence mapping from $L_{m}(\Phi)$ to $2^{W}$ by taking into account the modal operators $\square$ and $\diamond$. Based on the semantics of the modal propositional logic, the mapping $i$ still satisfies the properties (i1)-(i6). By combining equations (2) and (14), one can see that the following additional properties hold:

$$
\begin{aligned}
& i(\square \phi)=\underline{a p r}(i(\phi)), \\
& i(\diamond \phi)=\overline{\overline{a p r}}(i(\phi)) .
\end{aligned}
$$

This connection between rough-set operators and modal operators was also discussed by Chakraborty and Banerjee [5], Orlowska [19], and Pawlak [23].

In the modal logic system $S_{5}$, the necessity and possibility operators are characterized by the following axioms:

$$
\begin{aligned}
& \square(\phi \rightarrow \psi) \rightarrow(\square \phi \rightarrow \square \phi), \\
& \square \phi \rightarrow \phi, \\
& \phi \rightarrow \diamond \phi, \\
& \diamond \phi \rightarrow \square \diamond \phi, \\
& \diamond \square \phi \rightarrow \square \phi .
\end{aligned}
$$


From properties (i7) and (i8), and the property (R1) of rough-set model, it follows that:

$$
\begin{aligned}
i(\square \phi) & =\underline{\operatorname{apr}}(i(\phi)) \subseteq i(\phi), \\
i(\diamond \phi) & =\overline{\operatorname{apr}}(i(\phi)) \supseteq i(\phi) .
\end{aligned}
$$

Obviously, they correspond to axioms (M2) and (M3), where the logic implication is interpreted in terms of set inclusion. For example, for any world $w$, if $w \in \operatorname{apr}(i(\phi))$, i.e., $\square \phi$ is true, then $w \in i(\phi)$, i.e., $\phi$ is true. This means that (M2) is true in all possible worlds. Similarly, from (R9) and (R10) we can derive axioms (M4) and (M5). It should be noted that only the middle implication in (M1) is interpreted using set inclusion. It corresponds to the following property of the rough-set operators:

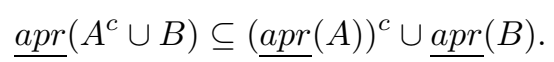

This can be easily proved based on the definition of the rough-set operators and property (R8). The correspondence between other properties of rough-set operators and modal operators can be easily established. Hence, our analysis clearly shows that the rough-set model provides a set-theoretic interpretation for modal logic. The standard logic connectives are characterized by the standard settheoretic operators, and the modal operators are characterized by the new set-theoretic operators in the rough-set model.

Probabilistic reasoning using rough-set modal can be formulated as follows. Suppose that the available information is only sufficient to define a probability function $P_{0}$ on Com(apr). Since not every proposition is mapped into a member of $\operatorname{Com}(a p r)$, it is impossible to define the probability for every proposition using equation (11). However, both $i(\square \phi)$ and $i(\diamond \phi)$ are composed sets in the approximation space $a p r=(W, \Re)$. We can therefore define a lower and an upper probability using $i(\square \phi)$ and $i(\diamond \phi)$ :

$$
\begin{aligned}
& P_{*}(\phi)=P_{0}(i(\square \phi)), \\
& P^{*}(\phi)=P_{0}(i(\diamond \phi)) .
\end{aligned}
$$

From (M2) and (M3), it follows that $P_{*}(\phi) \leq P(\phi) \leq P^{*}(\phi)$. In fact, the lower and upper probabilities thus defined are a pair of belief and plausibility functions [7, 8, 33, 34]. Therefore, reasoning using rough-set model is related to reasoning with belief and plausibility functions.

\section{$5 \quad$ Three-valued Logic and Interval-set Model}

Kleene's three-valued logic, denoted by $\mathrm{K}_{3}$, extends standard logic in a different direction [10, 29]. Instead of introducing more logic operators, $\mathrm{K}_{3}$ uses a third truth value $I$. This means that a proposition may in fact be true or false, but it is merely unknown or undeterminable what its specific truth status may be. With this new truth value, the semantics of logic connectives are redefined by the following truth tables:

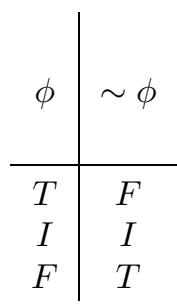

\begin{tabular}{l|c|c|c} 
& $\phi \wedge \psi$ & $\phi \vee \psi$ & $\phi \rightarrow \psi$ \\
\hline$\psi$ & $T I F$ & $T I F$ & $T I F$ \\
$\phi$ & & & \\
\hline$T$ & $T I F$ & $T T T$ & $T I F$ \\
$I$ & $I I F$ & $T I I$ & $T I I$ \\
$F$ & $F F F$ & $T I F$ & $T T T$
\end{tabular}

The symbols $T$ and $F$ stand for true and false, respectively. According to this definition, $\mathrm{K}_{3}$ satisfies properties similar to (I1)-(I9) of the interval-set model. In fact, one can obtain a set of properties for $K_{3}$ by replacing interval sets with propositions, $\neg$ with $\sim, \sqcap$ with $\wedge$, and $\sqcup$ with $\vee$. Thus, the interval-set model provides a possible-worlds based interpretation for $K_{3}$ in the same way as set theory provides for the two-valued logic. This point is explicated below.

The propositional language in Kleene's three-valued logic is the same as in standard propositional logic. The valuation mapping is modified to include the third truth value. For a possible world $w \in W$, we define a mapping $\pi_{w}: L(\Phi) \longrightarrow\{T, I, F\}$. By $\pi_{w}(\phi)=T$, we mean that the proposition $\phi$ is true in the world $w$, and $\pi_{w}(\phi)=F$ for case that the proposition is false. The third value $\pi_{w}(\phi)=I$ indicates that the truth value of $\phi$ is unknown or undeterminable. With this extended valuation function, we define two mappings, $i_{*}: L(\Phi) \longrightarrow 2^{W}$ and $i^{*}: L(\Phi) \longrightarrow 2^{W}$, as follows:

$$
\begin{aligned}
i_{*}(\phi) & =\left\{w \in W \mid \pi_{w}(\phi)=T\right\}, \\
i^{*}(\phi) & =\left\{w \in W \mid \pi_{w}(\phi) \in\{T, I\}\right\} .
\end{aligned}
$$


Obviously, we have $i_{*}(\phi) \subseteq i^{*}(\phi)$. Hence, $\left[i_{*}(\phi), i^{*}(\phi)\right]$ forms an interval-set representation of proposition $\phi$. The set $i^{*}(\phi)-i_{*}(\phi)$ denotes the set of possible worlds in which the truth value of $\phi$ is unknown or undeterminable, which is similar to the doubtful region in the rough-set theory [21].

With the possible-worlds semantics of $K_{3}$, we derive the following correspondence between $K_{3}$ logical operators and the interval-set operators:

$$
\begin{aligned}
& {\left[i_{*}(\sim \phi), i^{*}(\sim \phi)\right]=\neg\left[i_{*}(\phi), i^{*}(\phi)\right],} \\
& {\left[i_{*}(\phi \wedge \psi), i^{*}(\phi \wedge \psi)\right]=\left[i_{*}(\phi), i^{*}(\phi)\right] \sqcap\left[i_{*}(\psi), i^{*}(\psi)\right],} \\
& {\left[i_{*}(\phi \vee \psi), i^{*}(\phi \wedge \psi)\right]=\left[i_{*}(\phi), i^{*}(\phi)\right] \sqcup\left[i_{*}(\psi), i^{*}(\psi)\right],} \\
& {\left[i_{*}(\phi \rightarrow \psi), i^{*}(\phi \rightarrow \psi)\right]=\neg\left[i_{*}(\phi), i^{*}(\phi)\right] \sqcup\left[i_{*}(\psi), i^{*}(\psi)\right] .}
\end{aligned}
$$

With respect to negation, conjunction and disjunction, Kleene's three-valued logic is the same as the three-valued logic $\mathrm{L}_{3}$ of Eukasiewicz [29]. Hence, the interval-set model also provides an interpretation for $\mathrm{L}_{3}$.

Inference using interval-set model (i.e., Kleene's three-valued logic) can be carried out in a similar manner as in incidence calculus. For analyzing consistent possible worlds, we extend Nilsson's binary semantic tree into ternary semantic tree by adding the third truth value $I$. For the modus ponens rule, we derive the following consistent possible worlds:

\begin{tabular}{ccc}
$\phi \rightarrow \psi$ & $\phi$ & $\psi$ \\
\hline$T$ & $T$ & $T$ \\
$T$ & $I$ & $T$ \\
$T$ & $F$ & $\{T, I, F\}$ \\
$I$ & $T$ & $I$ \\
$I$ & $I$ & $\{I, F\}$ \\
$F$ & $T$ & $F$
\end{tabular}

For example, the second row says that if $\phi \rightarrow \psi$ is true $(T)$ and $\phi$ is unknown or undeterminable $(I)$ in a world $w$, according to $\mathrm{K}_{3}$, it is consistent to assume that $\psi$ is true $(T)$ in $w$. On the other hand, as indicated by the third row, if $\phi \rightarrow \psi$ is true $(T)$ and $\phi$ is false $(F)$ in $w$, then $\psi$ may take any of the three truth values $\{T, I, F\}$. By examining this table carefully, we can see that the interval set $\left[i^{*}(\phi) \cap i_{*}(\phi \rightarrow \psi), i^{*}(\phi \rightarrow \psi)\right]$ is the best possible bounds for interval set $\left[i_{*}(\psi), i^{*}(\psi)\right]$. Thus, we introduce the following modus ponens rule for the interval-set model:

$$
\frac{\left[i_{*}(\phi \rightarrow \psi), i^{*}(\phi \rightarrow \psi)\right], \quad\left[i_{*}(\phi), i^{*}(\phi)\right]}{i^{*}(\phi) \cap i_{*}(\phi \rightarrow \psi) \subseteq i_{*}(\psi) \subseteq i^{*}(\psi) \subseteq i^{*}(\phi \rightarrow \psi)},
$$

which is similar to the one used in incidence calculus.

Given a probability function $P_{0}$ defined on the set of all possible worlds $W$, a proposition $\phi$ will be associated with a pair of lower and upper probabilities:

$$
\begin{aligned}
P_{*}(\phi) & =P_{0}\left(i_{*}(\phi)\right), \\
P^{*}(\phi) & =P_{0}\left(i^{*}(\phi)\right) .
\end{aligned}
$$

In other words, an interval probability $\left[P_{*}(\phi), P^{*}(\phi)\right]$ is used to represent the truth of proposition $\phi$. However, this pair of lower and upper probabilities is not necessarily a pair of belief and plausibility functions. Using the extended modus ponens rule (19), we obtain a probabilistic version:

$$
\frac{P_{*}(\phi \rightarrow \psi) \leq P(\phi \rightarrow \psi) \leq P^{*}(\phi \rightarrow \psi), \quad P_{*}(\phi) \leq P(\phi) \leq P^{*}(\phi)}{P^{*}(\phi)+P_{*}(\phi \rightarrow \psi)-1 \leq P_{*}(\psi) \leq P(\psi) \leq P^{*}(\psi) \leq P^{*}(\phi \rightarrow \psi)},
$$

which may be regarded as a generalized result of equation (13). Thus, the interval-set model may be used to perform interval-probability inference [11].

Skowron proposed a generalized modus ponens rule using the notion of rough membership functions [32]. Since rough membership functions can be interpreted using probability functions, it is not surprising that similar results are obtained in the present study. As pointed out by Skowron, the bounds obtained using the generalized modus ponens rule cannot be improved further, provided that no further information is given. 


\section{Conclusion}

In this paper, we have provided a comparative study of the rough-set and interval-set models within the context of uncertain reasoning. The rough-set model is a generalization of set theory in which a pair of new set-theoretic operators are introduced. These operators are not truth-functional. On the other hand, the interval-set model is a generalization of set theory in which the standard set-theoretic operators are extended to interval sets. The extended operators are truth-functional. Within a framework of possible-worlds semantics analysis, we have explicitly shown that these two models correspond to two different extended propositional logics. The rough-set model generalizes set theory in the same way that the modal logic system $\mathrm{S}_{5}$ generalizes propositional logic, while the interval-set model extends set theory in the same way that Kleene's three-valued logic $\mathrm{K}_{3}$ extends propositional logic.

Reasoning approaches based on these two models have been examined in the framework of probabilistic reasoning. The rough-set model is related to reasoning using a special type of lower and upper probabilities known as belief and plausibility functions. An extended modus ponens rule has been suggested for reasoning using the interval-set model. It provides an useful tool for interval-probability reasoning.

In the present study, the discussion has been restricted to propositional logic systems. In the context of predicate logic systems, Lin, Liu and Yao, Nakamura, Rasiowa, and Rasiowa and Skowron have considered approximation logic systems using the notion of rough sets [13, 15, 25, 26, 27]. Recently, Nakamura and Matsueda proposed a new modal logic system R2 based on interval-set algebra [16]. It will be interesting to extend the results of this study in the light of these new developments.

\section{References}

[1] G. Alefeld, and J. Herzberger, Introduction to Interval Computations, New York, Academic Press, 1983.

[2] Z. Bonikowski, A certain conception of the calculus of rough sets. Notre Dame Journal of Formal Logic, 33, 412-421, 1992.

[3] Z. Bonikowski, Algebraic structures of rough sets. In: Rough Sets, Fuzzy Sets and Knowledge Discovery, W.P. Ziarko, Ed., London, Springer-Verlag, 242-247, 1994.

[4] A. Bundy, Incidence calculus: a mechanism for probabilistic reasoning. Journal of Automated Reasoning, 1, 263-283, 1985.

[5] M.K. Chakraborty, and M. Banerjee, Logic and algebra of rough sets. In: Rough Sets, Fuzzy Sets and Knowledge Discovery, W.P. Ziarko, Ed., London, Springer-Verlag, 196-207, 1994.

[6] B.F. Chellas, Modal Logic: An Introduction. Camberidge, Cambridge University Press, 1980.

[7] F. Correa da Silva, and A. Bundy, On some equivalence relations between incidence calculus and Dempster-Shafer theory of evidence. Proceedings of 6th International Workshop on Uncertainty in Artificial Intelligence, 378-383, 1990.

[8] D. Dubois, and H. Prade, Evidence measures based on fuzzy information. Automatica, 21, 547562,1985 .

[9] J.Y. Halpern, and Y. Moses, A guide to completeness and complexity for modal logics of knowledge and belief. Artificial Intelligence, 54, 319-379, 1992.

[10] S.C. Kleene, Introduction to Mathematics, New York, Groningen, 1952.

[11] H.E. Kyburg, Logical Foundations of Statistical Inference, Dordrecht, Reidel, 1974.

[12] T.Y. Lin, and Q. Liu, Rough Approximate Operators. In: Rough Sets, Fuzzy Sets and Knowledge Discovery, W.P. Ziarko, Ed., London, Springer-Verlag, 256-260, 1994.

[13] T.Y. Lin, Q. Liu, and Y.Y. Yao, Logic systems for approximate reasoning: via rough sets and topology. Methodologies for Intelligent Systems - 8th International Symposium, ISMIS'94, Lecture Notes in Artificial Intelligence 869, 65-74, 1994.

[14] R.E. Moore, Interval Analysis, Englewood Cliffs, New Jersey, Prentice-Hall, 1966. 
[15] A. Nakamura, On a logic of information for reasoning about knowledge. In: Rough Sets, Fuzzy Sets and Knowledge Discovery, W.P. Ziarko, Ed., London, Springer-Verlag, 186-195, 1994.

[16] A. Nakamura, and M. Matsueda, Rough logics based on incomplete knowledge systems. Proceedings of the Third International Workshop on Rough Sets and Soft Computing, 56-63, 1994.

[17] C.V. Negoiţă, and D.A. Ralescu, Applications of Fuzzy Sets to Systems Analysis, Basel, Birkhäuser Verlag, 1975.

[18] N.J. Nilsson, Probabilistic logic. Artificial Intelligence, 28, 71-87, 1986.

[19] E. Orlowska, Logical aspects of learning concepts. International Journal of Approximate Reasoning, 2, 349-364, 1988.

[20] E. Orlowska, Rough set semantics for non-classical logics. In: Rough Sets, Fuzzy Sets and Knowledge Discovery, W.P. Ziarko, Ed., London, Springer-Verlag, 143-148, 1994.

[21] Z. Pawlak, Rough sets. International Journal of Computer and Information Sciences, 11, 341-356, 1982.

[22] Z. Pawlak, Rough classification. International Journal of Man-Machine Studies, 20, 469-483, 1984.

[23] Z. Pawlak, Rough logic. Bulletin of the Polish Academy of Sciences Technical Sciences, 35, 253258, 1987.

[24] Z. Pawlak, Rough sets: a new approach to vagueness. In: L.A. Zadeh and J. Kacprzyk, Eds., Fuzzy Logic for the Management of Uncertainty, New York, John Wiley \& Sons, 105-118, 1992.

[25] H. Rasiowa, On approximation logics: a survey. Kurt Gödel Gesselschaft, Jahrbuch, 1990, 63-87, 1991.

[26] H. Rasiowa, and A. Skowron, Rough concepts logic. In: A. Skowron, Ed., Computation Theory, Lecture Notes in Computer Science, No. 208, Berlin, Springer-Verlag, 288-297, 1985.

[27] H. Rasiowa, and A. Skowron, Approximation logic. In: W. Bibel and K.P. Jantke, Eds., Mathematical Methods of Specification and Synthesis of Software Systems'85, Lecture Notes in Computer Science, No. 215, Berlin, Springer-Verlag, 123-139, 1985.

[28] S. Reeves, and M. Clarke, Logic for Computer Science. New York, Addison-Wesley, 1990.

[29] N. Rescher, Many-valued Logic, New York, McGraw-Hill, 1969.

[30] E.H. Ruspini, Approximate reasoning: past, present, future. Information Sciences, 57-58, 297313, 1991.

[31] E.H. Ruspini, On the semantics of fuzzy logic. International Journal of Approximate Reasoning, 5, 45-88, 1991.

[32] A. Skowron, Management of uncertainty in AI: a rough set approach. In: V.S. Alagar, S. Bergler and F.Q. Dong, Eds., Incompleteness and Uncertainty in Information Systems, London, SpringerVerlag, 69-86, 1994.

[33] A. Skowron, and J. Grzymala-Busse, From rough set theory to evidence theory. In: Advances in the Dempster-Shafer Theory of Evidence, R.R. Yager, M. Fedrizzi, and J. Kacprzyk, Eds., New York, John Wiley \& Sons, 193-236, 1994.

[34] P. Smets, Belief functions (with discussions). In: P. Smets, A. Mamdani, D. Dubois, and H. Prade, Eds., Non-standard Logics for Automated Reasoning, New York, Academic Press, 253-285, 1988.

[35] D. Vakarelov, A modal logic for similarity relations in Pawlak knowledge representation systems. Fundamenta Informaticae, XV, 61-79, 1991.

[36] Y.Y. Yao, Interval-set algebra for qualitative knowledge representation. Proceedings of the Fifth International Conference on Computing and Information, 370-374, 1993.

[37] Y.Y. Yao, and X. Li, Uncertain reasoning with interval-set algebra. In: Rough Sets, Fuzzy Sets and Knowledge Discovery, W.P. Ziarko, Ed., London, Springer-Verlag, 178-185, 1994. 